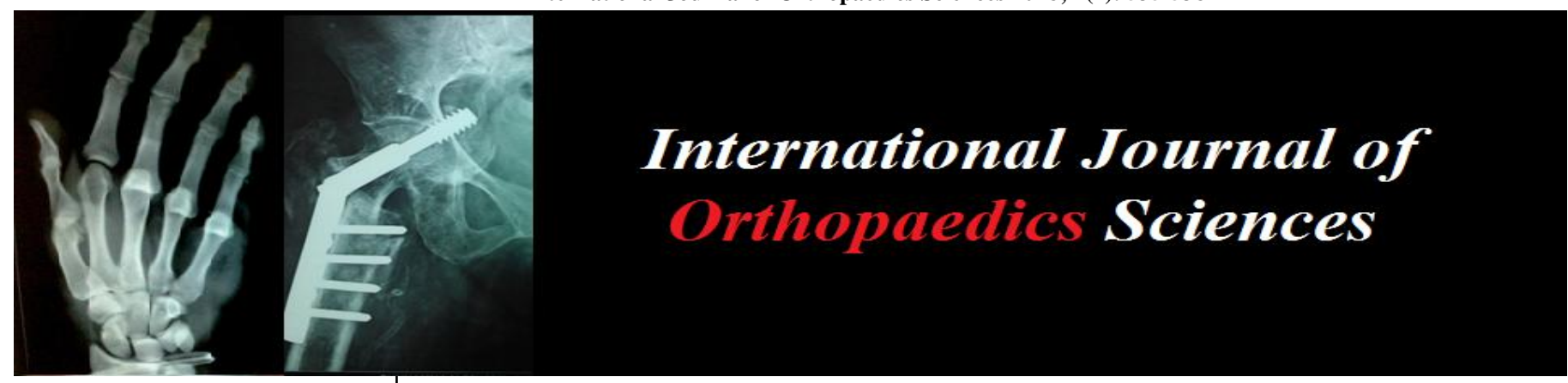

ISSN: $2395-1958$

IJOS 2018; 4(1): 535-538

(C) 2018 IJOS

www.orthopaper.com

Received: 28-11-2017

Accepted: 29-12-2017

Dr. Neelam Ramana Reddy Consultant, Department of Orthopaedics, Star Hospitals, Hyderabad, Telangana, India

Dr. Abhishek P Bhalotia Fellow, Department of Orthopaedics, Star Hospitals, Hyderabad, Telangana, India

Dr. Rajesh Podili

Consultant, Department of

Orthopaedics, Star Hospitals,

Hyderabad, Telangana, India

Dr. Naveen Malu

Fellow, Department of

Orthopaedics, Star Hospitals,

Hyderabad, Telangana, India
Correspondence

Dr. Abhishek P Bhalotia

Fellow, Department of

Orthopaedics, Star Hospitals,

Hyderabad, Telangana, India

\section{Impact of anterior stabilized insert on primary total knee arthroplasty (TKA) with an incompetent posterior cruciate ligament (PCL)}

\author{
Dr. Neelam Ramana Reddy, Dr. Abhishek Bhalotia, Dr. Rajesh Podili and \\ Dr. Naveen Malu
}

DOI: https://doi.org/10.22271/ortho.2018.v4.i1h.78

\begin{abstract}
Background: The role of posterior cruciate ligament (PCL) conservation and the choice of the level of constraint of polyethylene insert in total knee arthroplasty (TKA) are still debated. The use of anterior stabilized bearing has been associated with clinical success in a limited number of studies. We have used anterior stabilized bearing and studied its impact in case of lose or deficient PCL.

Material and Methods: A prospective study of 100 total knee arthroplasty to evaluate the impact of anterior stabilized bearing in partially or completely released PCL. Outcomes were measured with Knee Society scores, complications, revision TKA, and survival. Radiographs were analyzed for component alignment and evidence of loosening.

Results: Total 100 TKA were performed in seventy patients comprising of 53 females and 17 males. Mean follow-up in the study was 2.4 years (2-3 years). Knee Society Score for clinical and function scale increased from means of 54.2 (range, 36-68) and 48.6 (range, 33-62) preoperatively to 93.1 (range, 82100 ) and 90.4 (range, 76-100) at final follow-up respectively. Results were good or excellent in $93.5 \%$ of knees (79 excellent, 8 good); 6 patients reported fair results.

Conclusion: The use of an anterior stabilized (AS) bearing for PCL substitution has comparable clinical and radiographic results to traditional Cruciate Retaining (CR) and Posterior Stabilized (PS) TKA. These results suggest that this approach is an effective method to achieve stability without the PCL in primary TKA with the advantage of bone conservation.
\end{abstract}

Keywords: Total knee arthroplasty, anterior stabilized inserts, posterior cruciate ligament

\section{Introduction}

Total Knee Arthroplasty (TKA) is one of the most successful surgeries performed in last few decades with excellent functional results and enhanced recovery. This has been true in spite of controversies related to surgical approaches, huge variety of implants and different perioperative management protocols. The role of posterior cruciate ligament (PCL) conservation and the choice of the level of constraint of polyethylene insert in total knee arthroplasty (TKA) are still debated. The PCL is the primary restraint to posterior translation of the tibia throughout the range of knee flexion, and it acts as a primary stabilizer of the knee [1]. There are a variety of ways for managing the PCL in TKA, including PCL retaining (CR) or PCL sacrifice i.e, posterior stabilized (PS).

Proponents of PCL retention (CR) believes that PCL retention preserves more normal knee kinematics, along with reduced patellar complications, less constrain, improved stair-climbing ability and proprioceptive function ${ }^{[2-5]}$. Nevertheless, retention is not always an option and substitution of the PCL may be either required or preferred. PCL substitution in primary TKA has traditionally relied on a cam and post (cam-post) mechanisms in the form of a posteriorstabilized (PS) design. The advantages are increased range of motion (ROM), minimize premature wear of the polyethylene component and avoid compromised knee kinematics. Among the disadvantages of PCL-substituting systems are the need for additional resection of femoral bone from the intercondylar notch, potential wear at the femoral cam-polyethylene post interface, and abnormal gait patterns. Short and long-term evaluations of PS and CR knees have failed to show clinically significant differences in their outcomes ${ }^{[6-12]}$. 
The ultra-congruent inserts with higher anterior lip is designed for surgeons who want to sacrifice the posterior the posterior cruciate ligament but prefer not to substitute for it with a traditional cam and post mechanism as in a posterior stabilized knee ${ }^{[13-15]}$. The increased anterior lip height and decreased radius of curvature provides the necessary constraint and stability for a cruciate sacrificing procedure without the use of a post and cam mechanism. Advantages for the use of this design include ease and speed of conversion to substitute for the PCL using CR knee design, bone preservation resulting from the lack of need to resect intercondylar notch bone, eliminating cam and post impingement and the potential wear sequele and reducing the potential for patellar clunk ${ }^{[16,17]}$. Given these advantages and several studies in the last decade using this design we have used these inserts if PCL is incompetent or unable to balance, and assessed the early functional outcome for instabilities, range of motion and knee society score.

\section{Material and Methods}

After taking necessary approval from the institutional ethics committee, a prospective study of 100 TKA in 70 patients was performed. All patients had undergone primary TKA with an anterior-stabilized bearing between 2014 and 2015. There were significantly more $(\mathrm{p}=0.005)$ females $(\mathrm{n}=305)$ than males $(\mathrm{n}=163)$.

All TKAs were performed through a standard medial parapatellar approach with a single FDA-approved implant type (PFC Sigma, Depuy) by a senior author. The goal for coronal alignment was 5 degrees valgus (anatomic axis) and 0 degrees mechanical axis. The tibia cut was neutral to the mechanical axis in the coronal plane and with 3 to 5 degrees of posterior slope using an extramedullary guide. An initial medial release of the deep medial collateral ligament to the mid-sagittal line of the tibia was performed as part of the initial exposure. After bone preparation with measured resection technique, the symmetry of the flexion and extension gaps was evaluated with trial components in situ. Further medial, lateral, and PCL selective soft tissue release was performed to achieve balanced rectangular gaps. We don't do patellar resurfacing with CR implant so it was not done in any of the cases in this study.

Once trial implants were placed, the PCL was evaluated for both competence and balance. With the knee at 90 degrees of flexion using Pull out \& Lift off (POLO) test, the PCL was considered intact and balanced with a negative posterior drawer test and a tibiofemoral contact point at the middle of the tibial bearing. If these conditions were not satisfied, the PCL was considered either incompetent or excessively tight. If the PCL was considered incompetent or overly tight (requiring subsequent PCL recession or release), an anteriorstabilized bearing with 0 degrees inherent posterior slope and 9 to $11 \mathrm{~mm}$ anterior lip (size-dependent) was chosen as the bearing surface (Figure 1). All patients had the same postoperative protocol and follow-up schedule. Clinical outcomes were graded using the Knee Society Score (KSS), flexion range and signs of instabilities at 1 month, 3 months, 6 months and 1 year postoperatively and yearly thereafter. The last available KSS was used as the postoperative score for analysis.

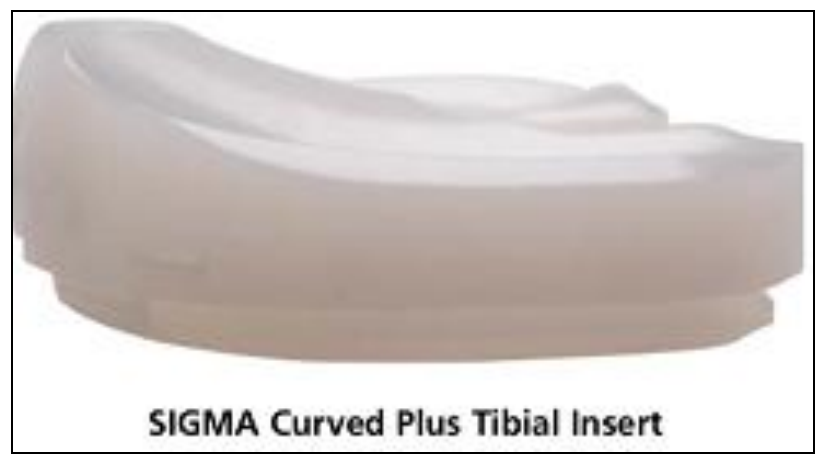

Fig 1: Sigma Curved Plus Anterior Stabilized Insert.

\section{Results}

The study includes hundred anterior stabilized (AS) total knee arthroplasty in 70 patients (30 bilateral and 40 unilateral TKA) comprised of 53 females and 17 males. Mean age at the time of surgery was 67.4 years (range, 54-89 years); mean BMI was 32 (range, 22-37). All surgeries were performed for the primary diagnosis of Osteoarthritis. Out of 100 TKA, 58 were right and 42 were left side. Seven knees in 5 patients (7\%) were lost to follow-up. Minimum follow-up criteria were 2 years and the mean follow-up in the study was found to be 2.4 years (2-3 years).

PCL was either partially or completely released depending upon intraoperative findings (Table 1). Instability was evaluated in all patients during each follow-up visit and was not found significant in any of the patient. Flexion range was found to increase from the mean preoperative range of 84-120 degrees to mean postoperative range of 92- 128 degrees. Knee Society Score for clinical and function scale increased from means of 54.2 (range, 36-68) and 48.6 (range, 33-62) to 93.1 (range, 82-100) and 90.4 (range, 76-100), respectively (Table 2 ). Results were good or excellent in $93.5 \%$ of knees (79 excellent, 8 good); 6 patients reported fair results. Radiographically, we could not see changes in prosthetic alignment or position but it should not be commented as the mean follow-up is very less to assess it. There was no gross evidence of thinning or asymmetry consistent with wear or failure of the tibial or femoral components. Complications in the form of patella-femoral pain were seen in 2 patients, morbidity in 1 patient with pulmonary embolism which was managed medically without any other complications (Table 3 ). Revision was not required in any of the patient but it would be difficult to comment with a short follow-up we had in the study.

Table 1: PCL status in patients undergoing Anterior Stabilized (AS) TKA

\begin{tabular}{|c|c|}
\hline Posterior Cruciate Ligament (PCL) Status & Anterior Stabilized (AS) TKA (N=100) \\
\hline Released Partially & 58 \\
\hline Released Completely & 42 \\
\hline
\end{tabular}

Table 2: Knee Society Score of the patients preoperatively, postoperatively along with mean improvement

\begin{tabular}{|c|c|}
\hline Knee Society Score (KSS) & Anterior Stabilized (AS) TKA \\
\hline Preoperative KSS & \\
\hline Clinical & $54.2(36-68)$ \\
\hline
\end{tabular}




\begin{tabular}{|c|c|}
\hline Functional & $48.6(33-62)$ \\
\hline Postoperative KSS & \\
\hline Clinical & $93.1(82-100)$ \\
\hline Functional & $90.4(76-100)$ \\
\hline Mean Improvement & 38.9 \\
\hline Clinical & 41.8 \\
\hline Functional & \\
\hline
\end{tabular}

Table 3: Complication Rate for Anterior Stabilized TKA

\begin{tabular}{|c|c|}
\hline Complication & Number of patients (\%) \\
\hline Infection & Nil \\
\hline Instability & Nil \\
\hline Aseptic Loosening & Nil \\
\hline Patello-Femoral Pain & $2(2.15 \%)$ \\
\hline Periprosthetic Fracture & Nil \\
\hline Revision & Nil \\
\hline Morbidity & $1(1.07 \%)$ \\
\hline
\end{tabular}

\section{Discussion}

The hypothesis of this article was an anterior-stabilized bearing provides adequate stability in presence of partially or completely released PCL. Reported advantages of the anterior-stabilized design include ease of conversion from CR to PCL substitution, bone preservation, and the potential for improved wear characteristics resulting from the increased surface contact area and potentially decreased surface contact forces ${ }^{[16,17]}$. Critics of anterior-stabilized bearings report their inability to restore normal knee kinematics and the potential to create a kinematic conflict ${ }^{[18,19]}$. Few, although generally favorable, clinical outcomes have been reported in the literature with the use of the anterior-stabilized bearing designs $[1,14,16,17]$. We sought to assess anterior-stabilized TKAs in terms of KSS, radiographic results, the frequency of complications (in particular manipulations for knee stiffness), revisions, and survival.

Hofmann et al., retrospectively reviewed 53 primary and 47 revision PCL-substituting TKAs using an anterior-stabilized polyethylene insert with a mean 60-month follow-up period and compared their results with 100 age and sex-matched patients who received TKA with a CR design ${ }^{[16]}$. They found no significant difference between the two groups in regard to the modified Hospital for Special Surgery score or ROM. Sathappan et al., similarly demonstrated good implant survival of $95 \%$ at 10 years with the use of an anteriorstabilized bearing design ${ }^{[17]}$. Laskin et al., found favorable outcomes with the use of an anterior-stabilized bearing design in a prospectively randomized trial of 176 patients with osteoarthritis using either a posterior-stabilized bearing or a deep-dished, anterior-stabilized tibial bearing after PCL sacrifice and found no statistically significant difference in ROM, ascending or descending stairs, pain, KSS, or stability ${ }^{[14]}$. Scott et al., reported similar clinical outcomes and no differences in the incidence of radiolucent lines when comparing anterior-stabilized inserts with posterior-lipped inserts ${ }^{[15]}$. We found no differences in the frequency of complications between the two groups, but we did identify that CR knees underwent revisions more frequently than did the anterior-stabilized TKAs, mainly related to instability.

Although the debate over the optimal method of management of the PCL is still not over with supporting literature for all the varied options, we found comparable knee society scores, complication rate, revision and survival with the use of an anterior-stabilized bearing when compared with a CR design. Because of the shorter follow-up we could not investigate long-term issues such as polyethylene wear and detailed kinematic such as quadriceps force during stair climbing, we could not strongly recommend the use of anterior-stabilized bearings based only on the results of this study. Anteriorstabilized bearings are attractive because it gives the option of using a CR femoral component in case of lose or deficient PCL without any additional bone cuts thus helps in preserving the bone stock. The major limitation of the study is short follow-up, operator bias on intraoperative selection of AS bearing and small sample size.

\section{Conclusion}

Anterior stabilized inserts were recently introduced basically to avoid the cam-mechanism wear and femoral inter-condylar bone sacrifice related to PS inserts. According to the available literature and our experience in this study, the functional and radiological outcomes of anterior stabilized inserts are comparable to other options at mid-term follow-up with advantage of bone conservation and less patellar complications.

We conclude that AS bearing is a viable option in presence of lose or deficient PCL and provides adequate stability and functional outcome. We need a larger sample size, multicenter trial and longer follow-up to see for complication rate, revision rate and survival.

\section{References}

1. Harner CD, Xerogeanes JW, Livesay GA, Carlin GJ, Smith BA, Kusayama $\mathrm{T}$ et al. The human posterior cruciate ligament complex: an interdisciplinary study. Ligament morphology and biomechanical evaluation. Am J Sports Med. 1995; 23:736-745.

2. Andriacchi TP, Galante JO, Fermier RW. The influence of total knee-replacement design on walking and stairclimbing. J Bone Joint Surg Am. 1982; 64:1328-1335.

3. Ewald FC, Jacobs MA, Miegel RE, Walker PS, Poss R, Sledge CB. Kinematic total knee replacement. J Bone Joint Surg Am. 1984; 66:1032-1040.

4. Kelman GJ, Biden EN, Wyatt MP, Ritter MA, Colwell $\mathrm{CW}$ Jr. Gait laboratory analysis of a posterior cruciatesparing total knee arthroplasty in stair ascent and descent. Clin Orthop Relat Res. 1989; 248:21-25; discussion 2526.

5. Schultz RA, Miller DC, Kerr CS, Micheli L. Mechanoreceptors in human cruciate ligaments. A histological study. J Bone Joint Surg Am. 1984; 66:10721076.

6. Andriacchi TP, Galante JO. Retention of the posterior cruciate in total knee arthroplasty. J Arthroplasty. 1988; 3:S13-S19.

7. Lozano-Calderon SA, Shen J, Doumato DF, Greene DA, Zelicof SB. Cruciate-Retaining vs posterior-substituting inserts in total knee arthroplasty: functional outcome comparison. J Arthroplasty. 2013; 28:234-242.e1.

8. Parsley BS, Conditt MA, Bertolusso R, Noble PC. Posterior cruciate ligament substitution is not essential for excellent postoperative outcomes in total knee arthroplasty. J Arthroplasty. 2006; 21:127-131. 
9. Shoji H, Wolf A, Packard S, Yoshino S. Cruciate retained and excised total knee arthroplasty: a comparative study in patients with bilateral total knee arthroplasty. Clin Orthop Relat Res. 1994; 305:218-222.

10. Tanzer M, Smith K, Burnett S. Posterior-stabilized versus cruciate retaining total knee arthroplasty. J Arthroplasty. 2002; 17:813-819.

11. Victor J, Banks S, Bellemans J. Kinematics of posterior cruciate ligament-retaining and substituting total knee arthroplasty: a prospective randomised outcome study. J Bone Joint Surg Br. 2005; 87:646-655.

12. Wing CK, Kwok-Hing C. Sixteen years' result of posterior-stabilized TKA. J Knee Surg. 2012; 25:245-248.

13. Font-Rodriguez DE, Scuderi GR, Insall JN. Survivorship of cemented total knee arthroplasty. Clin Orthop. 1997, 79.

14. Laskin RS, Maruyama Y, Villaneuva M et al. Deep dish congruent tibial component use in total knee arthroplasty: a randomized prospective study. Clin Orthop, 2000, 36.

15. Scott RD, Thornhill TS. Posterior cruciate supplementing total knee replacement using conforming inserts and cruciate recession. Effect on range of motion and radiolucent lines. Clin Orthop. 1994, 146.

16. Hofmann AA, Tkach TK, Evanich CJ, Camargo MP. Posterior stabilization in total knee arthroplasty with use of an ultracongruent polyethylene insert. J Arthroplasty. 2000; 15:576-583.

17. Sathappan SS, Wasserman B, Jaffe WL, Bong M, Walsh M, Di Cesare PE. Midterm results of primary total knee arthroplasty using a dished polyethylene insert with a recessed or resected posterior cruciate ligament. J Arthroplasty. 2006; 21:1012-1016.

18. Daniilidis K, Skwara A, Vieth V, Fuchs-Winkelmann S, Heindel W, Stuckmann V, Tibesku CO. Highly conforming polyethylene inlays reduce the in vivo variability of knee joint kinematics after total knee arthroplasty. Knee. 2012; 19:260-265.

19. Dennis DA, Komistek RD, Colwell Jr CE, Ranawat CS, Scott RD, Thornhill TS, Lapp MA. In vivo anteroposterior femorotibial translation of total knee arthroplasty: a multicenter analysis. Clin Orthop Relat Res. 1998; 356:47-57. 